\title{
Endobronchial ultrasound-guided transbronchial needle aspiration under conscious sedation with meperidine and midazolam
}

\author{
Lorenzo Agostini1, Nicola Facciolongo1, Mirco Lusuardi2, Eleonora Casalini1, Carla Galeone1, \\ Luciano Lasagni', Luigi Zucchi1
}

1 Pulmonology Unit, Department of Cardio-Thoracic-Vascular Surgery and Critical Care Medicine,
Azienda Ospedaliera ASMN-Istituto di Ricovero e Cura a Carattere Scientifico, Reggio Emilia
2 Respiratory Rehabilitation, AUSL Reggio Emilia, S. Sebastiano Hospital, Correggio (RE), Italy

\begin{abstract}
Endobronchial ultrasound guided transbronchial needle aspiration (EBUS-TBNA) is usually performed under general anesthesia or deep sedation with drugs such as Propofol that, at least in Italy, can be administered only by an anesthesiologist. Aim of the study was to assess conscious sedation feasibility, safety and tolerability using meperidine and midazolam as administered by Pulmonologist and relevant impact on the efficiency of the sampling procedures.
\end{abstract}

Corresponding author: Nicola Facciolongo, Pulmonology Unit, Department of Cardio-Thoracic- Vascular Surgery and Critical Care Medicine, Azienda Ospedaliera ASMN, Istituto di Ricovero e Cura a Carattere Scientifico, Via Risorgimento 81, 42100 Reggio Emilia, Italy.

Tel. +39.0552.296479 - Fax: +39.0522.296182.

E-mail: facciolongo.nicola@asmn.re.it

Key words: Endobronchial ultrasound guided transbronchial needle aspiration (EBUS-TBNA); conscious sedation; midazolam; meperidine.

Contributions: LA, NF, study concept and design, study coordination, recruitment of patients, manuscript writing and review, results interpretation; CG, EC, subjects enrolling, manuscript writing, data acquisition; ML, clinical data analysis, project supervision, manuscript writing and review; LL, recruitment of patients, results interpretation, deep sedation performing; LZ, recruitment of patients, results interpretation and review. All authors reviewed the manuscript and approved the final version.

Conflict of interest: the authors declare no conflict of interest.

Acknowledgments: We thank our nurse team composed by Manuela Quartaroli, Fiorella Noci and Simona Devoti, for their invaluable contribution to our patients' care and their support to our protocol of sedation management.

Received for publication: 29 August 2016

Accepted for publication: 13 March 2017

(C) Copyright L. Agostini et al., 2017

Tipografia PI-ME Editrice, Italy

Monaldi Archives for Chest Disease 2017; 87:768

doi: 10.4081/monaldi.2017.768

This article is distributed under the terms of the Creative Commons Attribution Noncommercial License (by-nc 4.0) which permits any noncommercial use, distribution, and reproduction in any medium, provided the original author(s) and source are credited.
All patients undergoing EBUS-TBNA from February 2013 to July 2014 were examined retrospectively. Efficiency using Meperidine and Midazolam during EBUS-TBNA has been assessed: completion of lymphnodal sampling, sampling adequacy, diagnostic yield, cough during endoscopic procedure complications and need for procedure repetition with Anesthesiology assistance. Patient satisfaction and cost/effectiveness were also evaluated. One hundred and thirty-four consecutive patients were considered; $97.7 \%$ completed the procedure. In $96.9 \%$ of cases the prefixed program of lymph-nodal sampling was accomplished. Sampling adequacy was $92,4 \%$. Diagnostic yield was $55 \%$. In $94.7 \%$ of cases cough was absent or did not interfere with EBUS-TBNA. The need to repeat the endoscopic procedure occurred in 6 cases but only in 2 the presence of an Anesthesiologist was required. Patient satisfaction was very high, with $95.9 \%$ of subjects reporting they would "definitely return". A $27 \%$ cost reduction was calculated. EBUS-TBNA under conscious sedation using Meperidine and Midazolam prescribed and administered by pulmonologist without the Anesthesiologist assistance, revealed to be a safe, well tolerated and cost saving procedure. The efficiency of sampling was good, apart from a relatively low diagnostic yield due to different expertise of operators.

\section{Introduction}

Endobronchial ultrasound guided transbronchial needle aspiration (EBUS-TBNA) is a minimally invasive well-established technique for mediastinal and hilar lymph node or mass diagnosis [1-3] and for lung cancer staging [4] that allows a tissue sampling more accurate than blind transbronchial needle aspiration (TBNA) [5].

The size of the EBUS-TBNA instrumentation requires oral introduction that is associated with low patient satisfaction and tolerability [6]; as a consequence, EBUS-TBNA has been traditionally performed under general anesthesia or deep sedation $[7,8]$ with drugs such as Propofol that, at least in Italy, have to be administered by an Anesthesiologist $[9,10]$. Due to a general shortage of health resources, in particular qualified health professionals, the present number of Anesthesiologists is hardly sufficient to guarantee assistance during all invasive diagnostic procedures. In the setting of gastro-intestinal endoscopy, effective sedation protocols have been developed that can be administered and monitored without anesthesiology support, inducing only a level of sedation defined as moderate or conscious, allowing the patient to maintain ventilatory and cardiovascular function and in the same time the ability to make purposeful responses to verbal or tactile stimulation [11]. One of these protocols combines the sedative and amnesic properties of midazolam, a short-acting benzodiazepine, with the antitussive and analgesic properties of meperidine, an opioid agent $[9,10]$. The possibility for the pulmonologist to work without 
the assistance of other specialists would allow an optimization in the use of human resources in a period of anesthesiologist shortage.

This study aimed to assess conscious sedation feasibility, safety and tolerability using meperidine and midazolam as administered by pulmonologist and relevant impact on the efficiency of the sampling procedures.

\section{Materials and Methods}

The study was a retrospective analysis of data of all the patients that from February 2013 to July 2014 underwent EBUS-TBNA at our hospital for diagnosis and/or staging of mediastinal or hilar lymphadenopathy or paratracheal or peribronchial mass (PET positive or with a short axis $\geq 1$ $<3 \mathrm{~cm}$ ). A criterion of selective sampling of suspicious nodes only was adopted, corresponding to grade $\mathrm{C}$ in the classification of thoroughness levels in American College of Chest Physicians guidelines [12].

The primary endpoint was the completion of the prefixed lymphnodal sampling.

Secondary endpoints were:

1) the sampling adequacy, defined as specimens demonstrating presence of lymph-nodal tissue or lymphocytes with or without atypical cells

2) diagnostic yield (number of procedures leading to a definite diagnosis/total number of procedures); definite diagnoses were considered as the sum of true positive and true negative cases (confirmed by mediastinoscopy or surgery or imaging follow up at 2 years).

3) complications occurring before or during EBUS-TBNA

4) need for repetition of the endoscopic procedure

5) patient satisfaction

6) cost effectiveness

Local institutional review board approved this study; all patients had signed a formal informed consent to the procedure and to the possible use of data for research.

EBUS-TBNA was performed alternatively by one of the four specialists of our endoscopic unit. The period on evaluation (2013-2014) included the early phase of the learning curve with bronchoscopists having a different level of expertise on EBUS-TBNA. Patients undergoing EBUS-TBNA were either hospitalized or outpatients. Topical anesthesia was administered with Lidocaine $2 \%$ [9]. Conscious sedation was obtained with intravenous (i.v.) Meperidine and Midazolam. In particular, patient received an initial i.v. bolus of $50 \mathrm{mg}$ Meperidine. Supplement of $10 \mathrm{mg}$ Meperidine, if necessary, were administered in order to achieve a total maximum dosage of $1 \mathrm{mg} / \mathrm{Kg}$. An initial Midazolam $2.5 \mathrm{mg}$ i.v. bolus was followed by bolus of $1 \mathrm{mg}$ in order to obtain an adequate patient sedation, considering a maximum dosage of 0.1 $\mathrm{mg} / \mathrm{Kg}[9,10)$. EBUS-TBNA was performed with real-time ultrasound biopsy bronchoscope (BF-UC180F OL8, Olympus, Tokyo, Japan) 7,5 $\mathrm{MHz}$ linear ultrasound transducer was linked to a processor. Transbronchial needle biopsies were performed with a dedicated 22 gauge needle (NA 201SX-4022 Olympus, Tokyo, Japan).

With the patient on a supine position, the bronchoscope was introduced through the mouth during the induction of conscious sedation administered by an assisting nurse [10[. Oxygen saturation, ECG, heart rate, blood pressure, vital signs and the thoracic cage excursion were monitored throughout the procedure according to bronchoscopy BTS guidelines [10[. Supplemental oxygen was administered as needed, in order to maintain a target $\mathrm{SaO}_{2}>94 \%$ In case of persistent desaturation the use of antagonists (flumazenil and naloxone for benzodiazepines and opioids, respectively) was considered. The anesthesiologist was quickly available in case of emergency. At the conclusion of the procedure, the patient was brought to the recovery room. Vital signs were monitored for at least $1 \mathrm{~h}$. Outpatients were discharged with an adult responsible for driving the patient home, and with a recommendation to avoid driving for the following $24 \mathrm{~h}$. After the procedure flumazenil was administered to all outpatients. Sampling was always started from the lymph-nodal station entailing the most advanced staging: we initiated at N3 regions followed by N2 and N1 regions according to the recent guidelines for acquisition and preparation of EBUS-TBNA specimens [12]. At our institution, cytology Rapid On Site Evaluation (ROSE) in not usually available [14].

The duration time of the procedure and the number of passes were registered. At the end of the procedure a questionnaire on cough was administered to the patient by the bronchoscopist or an assisting nurse. Two hours later, the patient filled in a 5 point Likert scale on the acceptance and willingness to repeat the procedure. A cost evaluation comparing the 2 different protocols (without and with anesthesiology assistance) was carried out using standard costs according to current administrative tables.

\section{Statistics}

Data descriptive of the study population are reported as mean \pm standard deviation (SD) or range as specified in text or tables.

\section{Results}

From February 2013 to July 2014, 134 consecutive patients underwent EBUS-TBNA during conscious sedation with meperidine and midazolam. Patients' characteristics and indications for EBUS-TBNA are reported in Table 1 .

Among 134 patients eligible for EBUS-TBNA, 131 (97.8\%) completed the procedure; in one patient a low peripheral oxygen saturation $\left(\mathrm{SpO}_{2}\right)$ induced to stop the exam, in two patients agitation occurred that was not controlled by Meperidine and Midazolam.

Procedural data on the 131 completing EBUS-TBNA is reported in Table 2.

As the primary endpoint: the lymph-nodal sampling was completed in $127(96.9 \%)$ out of 131 patients. For two of the four dropping out patients the endoscopist was not able to identify all the lymph-nodal stations according to CT/PET images; hemorrhage in a third case and tachyarrhythmia in the fourth induced to stop the endoscopic procedure before the sampling being completed. Sampling adequacy was $92.4 \%$ (121 out of 131 patients).

Table 1. Characteristics sof patients with an indication for EBUS-TBNA.

\begin{tabular}{ll}
\hline Male, n (\%) & $92(68.7)$ \\
Female, n (\%) & $42(31.3)$ \\
Age, years - mean (SD) & $64.7(13.3)$ \\
Weight, Kg - mean (SD) & $74.3(14.8)$ \\
Indication for EBUS-TBNA, n (\%) & \\
- Pleural or pulmonary disease and lymphadenopathy & $96(71.6)$ \\
- Isolated mediastinal lymphadenopathy & $30(22.4)$ \\
- Paratracheal or peribronchial mass (T) with or without & \\
$\quad$ mediastinal lymphadenopathy & $8(6)$ \\
- EBUS-TBNA, unplanned, n (\%) & $78(58.2)$ \\
- EBUS-TBNA, planned, n (\%) & $32(23.9)$ \\
- TBNA, n (\%) & $21(15.7)$ \\
- repeated EBUS-TBNA & $3(2.2)$ \\
\hline
\end{tabular}


Diagnostic yield: the most common diagnosis was malignancy (51/131 38.9\%), followed by sarcoidosis in 6 cases (4.6\%) and tuberculosis in one $(0.8 \%)$, globally accounting for a true positive yield of $44.3 \%$. Non-diagnostic sampling was $48.1 \%$ : in 13 cases (9.9\%) the presence of anthracotic lymph node fragments was observed and in 50 patients (38.2\%) the specimens showed lymphocytes with or without atypical cells.

Among 63 patients whose sampling was adequate but ineffective for diagnosis, evaluations continued in 26 cases (4 EBUS, 2 EUS, 20 surgery/mediastinoscopy): overall, 14 true negative and 12 false negative cases (6 lung cancer metastases, 3 lymphomas, 3 necrotizing granulomas) were observed. The sum of true positive and true negative cases (i.e., overall diagnostic yield) was 55\%. Adverse events before EBUSTBNA 3 adverse events were registered: in one case (0.7\%) a severe $\mathrm{SpO}_{2}$ desaturation soon after drug administration prevented to start the procedure; in 2 patients (1.7\%) agitation occurred that was not controlled by Meperidine and Midazolam.

During EBUS-TBNA adverse events occurred in 7 patients: five subjects (3.7\%) presented a transient tachyarrhythmia with spontaneous resolution; in one patient hemorrhage occurred after needle puncture; in one patient, a severe peripheral $\mathrm{SpO}_{2}$ desaturation rapidly resolved with Flumazenil and Naloxone administration.

Table 2. Procedural data on 131 patients completing EBUS-TBNA.

\begin{tabular}{ll}
\hline Procedure time, min - mean (SD) & $29(10.5)$ \\
Sedative doses, mg - mean (SD) & \\
- Midazolam, mg & $4.8(1.3)$ \\
- Meperidine, mg & $56.1(16.7)$ \\
Lymph node stations sampled, n (\%) & \\
-7 & $65(32.2)$ \\
$-4 \mathrm{R}$ & $56(27.7)$ \\
$-4 \mathrm{~L}$ & $22(10.9)$ \\
$-11 \mathrm{R}$ & $16(7.9)$ \\
$-10 \mathrm{~L}$ & $9(4.5)$ \\
$-2 \mathrm{R}$ & $8(3.9)$ \\
$-10 \mathrm{R}$ & $8(3.9)$ \\
$-11 \mathrm{~L}$ & $5(2.5)$ \\
$-12 \mathrm{R}$ & $3(1.5)$ \\
-3 & $1(0.5)$ \\
-1 & $1(0.5)$ \\
$-\mathrm{T}$ & $8(4)$ \\
Number of lymph node stations sampled & \\
per patient - mean (range) & $1.5(1-4)$ \\
Number of passes per patient, mean (range) & $3.6(1-7)$ \\
\hline
\end{tabular}

T, Paratracheal or parabronchial masses.
The need to repeat the endoscopic procedure with an anesthesiologist occurred only in two of the 134 subjects (1.5\%), in one case to perform EBUS-TBNA, in the second case to continue the diagnostic procedure with transbronchial biopsies.

Patient satisfaction in 121 out of 131 patients rated as very high: among the patients who answered to the questionnaire, 116 (95.9\% of 121) reported they would "definitely return" for EBUS-TBNA in the future if required, two patients would "probably" return, one was "unsure" and two answered that they "definitely would not return" for EBUS-TBNA.

Coughing in $94.7 \%$ of cases (124 out of 131 patients) was absent or did not interfere with EBUS-TBNA. In 7 patients (5.3\%) cough interfered with the sampling of lymph nodes and caused a significant delay of the endoscopic procedure.

The procedure carried out without anesthesiology assistance was associated with a cost reduction of $27 \%$ (Table 3 ).

\section{Discussion}

Moderate or conscious sedation during EBUS-TBNA permits the control of cough and movements providing a comfortable setting for the bronchoscopist and allowing to complete the endoscopic procedure in the shortest time as possible; at the same time conscious sedation has the advantage of preserving protective airway reflexes and pulmonary function, while permitting the patients to follow verbal instructions. We were also supported in our practice by the long experience of gastrointestinal endoscopy in using this kind of sedation, including our hospital [11], and by the absence in literature of clear evidence demonstrating a superior efficiency of general anesthesia or deep sedation during EBUS-TBNA [13, 15-17].

To our knowledge very few reports have been published evaluating the efficacy of Meperidine and Midazolam used alone or in combination during bronchoscopy and especially during EBUS-TBNA [18, 19). At present, there is not a single, well established method of anesthesia universally used during EBUS-TBNA.

In our experience EBUS-TBNA under moderate sedation with meperidine and midazolam seems to be an effective procedure, apart from a relatively low diagnostic yield. The lymph nodal sampling was not completed only in four cases $(3,1 \%)$ out of 131 patients. The sampling adequacy was $92.4 \%$ (121/131) in agreement with data previously published [17,20].

Among those 10 cases $(7,6 \%)$ with inadequate sampling eight occurred in the first year of our EBUS-TBNA experience so these data can be explained by limited skills during the early phase in the use of EBUS by our endoscopists. The importance of the learning curve in the implementation of endoscopic ultrasound application and parallel improvement of diagnostic yield have been clearly demonstrated [21].

Table 3. Cost variations comparing general anesthesia versus conscious sedation.

\begin{tabular}{|c|c|c|c|c|}
\hline & General anesthesia & Cost, $€$ & Moderate sedation & Cost, $€$ \\
\hline \multirow[t]{3}{*}{ Personnel } & 1 anesthesiologist $/ 1.5 \mathrm{~h}$ & 90 & & \\
\hline & 1 pulmonologist / $1.5 \mathrm{~h}$ & 90 & 1 pulmonologist $/ 1 \mathrm{~h}$ & 60 \\
\hline & 2 nurses / $1.5 \mathrm{~h}$ & 78 & 2 nurses / $1 \mathrm{~h}$ & 52 \\
\hline Disposables and drugs & & 239 & & 189 \\
\hline Equipment & & 950 & & 950 \\
\hline Indirect costs & & 180 & & 180 \\
\hline Hospitalization & & 336 & & 0 \\
\hline Total costs & & 1963 & & 1431 \\
\hline
\end{tabular}


A definite diagnostic yield (i.e. sum of true positive and true negative cases) was observed in a percentage (55\%) lower than several data in literature [22-25]. Better diagnostic performance was reported by Casal et al. (69\% diagnostic yield) but all the procedures were carried out by the same expert operator [24]. A diagnostic yield of $66 \%$ was recorded by Yarmus et al., but all operators had similar and advanced expertise [25]. A recent systematic review reports an overall diagnostic yield of $52.3-100 \%$ for deep sedation and of $46.1-85.7 \%$ for moderate sedation [26]. Another reason could be the need to perform a greater number of passes per lymph node as recommended by recent guidelines [13]). In our study, we cannot comment about diagnostic sensitivity because not all patients underwent surgery.

Patient satisfaction resulted very high: among the patients who answered to the questionnaire 116 (116/121 95.9\%) would "definitely return" for EBUS-TBNA in the future if required; only two patients answered that they "definitely would not return" for EBUS-TBNA. Our data prove that EBUS-TBNA under conscious sedation is associated with extremely high patient satisfaction, in agreement with previous reports, including studies that used different agents for conscious sedation [16,24,27].

A $94.7 \%$ of full cough control with conscious sedation is a very positive result and compares favorably with data in literature [27]. In any case, the retrograde amnesia due to midazolam always deleted the patient's memory of the discomfort linked to the cough.

About complications $[28,29]$ we overall registered a peripheral oxygen desaturation in two cases; however, the respiratory depression was always controlled by flumazenil and naloxone administration. As a matter of fact, all benzodiazepines can induce paradoxical effects such as anxiety, restlessness, mental confusion and psychomotor agitation. Tachyarrhythmia episodes that occurred in five patients were of short duration and spontaneously resolved. We assisted to an escalation in the level of care in only $1.5 \%$ of cases similarly to other data in literature [28].

In our setting the possibility to carry out the EBUS-TBNA procedure without an anesthesiologist facilitates the performance especially of the unplanned EBUS-TBNA examinations (e.g., when indication is given by evaluating CT few minutes before starting the procedure) that in our series amount to $58.2 \%$ of cases [30,31].

Conscious sedation had a significant impact on organization and direct costs since the procedure could be carried out efficiently and safely without anesthesiology assistance, with a calculated $27 \%$ reduction of the resources to be considered with the team including the anesthesiologist.

Among limitations of our study, we did not compare moderate and deep sedation during EBUS-TBNA, nevertheless we believe that our experience can further confirm that this procedure does not necessarily require propofol administration, general anesthesia or assisted breathing with intubation or laryngeal mask. Our results are also supported by the recent guidelines for the acquisition and preparation of EBUS-TBNA specimens $[13,24]$ underlining that the type of anesthesia seems to have no influence on the diagnostic yield of EBUS-TBNA. Furthermore, the same guidelines remark the importance of the number of aspirates per lymph node for the diagnostic yield of the specimen [13], and probably the low percentage of specific diagnosis in our study is due to the necessity to perform more passes for lymph node rather than to the type of sedation.

Anyway, the performance of EBUS-TBNA during moderate sedation does not exclude the possibility to resort to deep sedation or general anesthesia in a second time in case of failure.

A recent randomized trial on 149 patients is in agreement with our experience since it concluded that EBUS-TBNA performed under moderate sedation results in comparable diagnostic yield, rate of major complications, and patient tolerance as under general anesthesia [24].
In conclusion, EBUS-TBNA under conscious sedation using meperidine and midazolam prescribed by Pulmonologist without the Anesthesiologist assistance revealed to be a safe, well tolerated and cost saving procedure. The efficiency of sampling was good, apart from a relatively low diagnostic yield due to different expertise of operators in the first phase of the learning curve at our unit.

\section{References}

1. Nakajima T, Yasufuku K, Yoshino I. Current status and perspective of EBUS-TBNA. Gen Thorac Cardiovasc Surg 2013;61:390-6.

2. Tournoy K, Rintoul RC, van Meerbeeck JP, et al. EBUS-TBNA for diagnosis of central parenchymal lung lesions not visible at routine bronchoscopy. Lung Cancer 2009;63:45-49.

3. Rivera MP, Mehta AC, Wahidi MM. Establishing the diagnosis of lung cancer: diagnosis and management of lung cancer, 3rd ed. American College of Chest Physicians evidence-based clinical practice guidelines. Chest 2013; 143:e142S-e165S.

4. Kinsey CM, Arenberg DA. Endobronchial ultrasound-guided transbronchial needle aspiration for non-small cell lung cancer staging. Am J Respir Crit Care Med 2014;189:640-9.

5. Herth F, Becker HD, Ernst A. Conventional vs endobronchial ultrasound-guided transbronchial needle aspiration: a randomized trial. Chest 2004;125:322-5.

6. Lechtzin N, Rubin HR, White P Jr, et al. Patient satisfaction with bronchoscopy. Am J Respir Crit Care Med 2002;166:1326-31.

7. Sarkiss M, Kennedy M, Riedel B, et al. Anesthesia technique for endobronchial ultrasound-guided fine needle aspiration of mediastinal lymph node. J Cardiothorac Vasc Anesth 2007;21:892-6.

8. Kennedy MP, Shweihat Y, Sarkiss M, et al. Complete mediastinal and hilar lymph node staging of primary lung cancer by endobronchial ultrasound: Moderate sedation or general anesthesia? Chest 2008;134:1350-1.

9. Wahidi MM, Jain P, Jantz Met, al. American College of Chest Physicians consensus statement on the use of topical anesthesia, analgesia and sedation during flexible bronchoscopy in adult patients. Chest 2011;140:1342-50.

10. Du Rand IA, Blaikley J, Bootonet R, al. British Thoracic Societyguideline for diagnostic flexible bronchoscopy in adults. Thorax 2013;68:i1-i44.

11. American Society for Gastrointestinal Endoscopy Guideline. Sedation and anesthesia in GI endoscopy. Gastrointest Endosc 2008;68:815-26.

12. Silvestri GA, Gonzales AV, Jantz MA, et al. Methods for staging nonsmall cell lung cancer: Diagnosis and management of lung cancer, 3 rd ed. American College of Chest Physicians evidence-based clinical practice guidelines. Chest 2013;143:e211S-e250S.

13. van der Heijden EHFM, Casal RF, Trisolini R, et al. Guideline for the acquisition and preparation of conventional and endobronchial ultrasound-guided transbronchial needle aspiration specimens for the diagnosis and molecular testing of patients with known or suspected lung cancer. Respiration 2014;88:500-17.

14. Nakajima T, Yasufuku K, Saegusa F, et al. Rapid on-site cytologic evaluation during endobronchial ultrasound-guided transbronchial needle aspiration for nodal staging in patients with lung cancer. Ann Thorac Surg 2013;95:1695-99.

15. Hert FJL, Eberhardt R, Vilmann P, et al. Real-time endobronchial ultrasound guided transbronchial needle aspiration for sampling mediastinal lymph nodes. Thorax 2006;61:795-8.

16. Ferrando J, Fernandez-Navamuel I, Clemente CC, et al. Tolerance of the endobronchial ultrasound (EBUS) using five different sedation models. Chest 2014;145(3_Meeting Abstracts):492A. 
17. Ost DE, Ernst A, Lei X, et al. Diagnostic yield of endobronchial ultrasound-guided transbronchial needle aspiration. Results of the AQuiRE bronchoscopy registry. Chest 2011;140:1557-66.

18. Matot I, Kramer Mordechai R. Sedation in outpatient flexible bronchoscopy: Alfentanil-Propofol versus meperidine-midazolam. J Bronchol 1999;6:74-7.

19. Ando K, Ohkuni Y, Fukazawa M, et al. Sedation with meperidine for endobronchial ultrasound-guide transbronchial needle aspiration. J Broncho Intervent Pulmonol 2010;17:329-33.

20. Jernlås B, Nyberger $H$, Ek L, et al. Diagnostic yield and efficacy of endobronchial ultrasound-guided transbronchial needle aspiration in mediastinal lymphadenopathy. Clin Respir J 2012;6; 88-95.

21. Bellinger CR, Chatterjee AB, Adair N, et al. Training in and experience with endobronchial ultrasound. Respiration 2014;88:478-3.

22. Hirose T, Okuda K, Ishida H, et al. Patient satisfaction with sedation for flexible bronchoscopy. Respirology 2008;13:722-7.

23. Goyal G, Pisani MA, Murphy TE, et al. Advanced diagnostic bronchoscopy using conscious sedation and the laryngeal nerve block: Tolerability, thoroughness, and diagnostic yield. Lung 2014; 192:905-13.

24. Casal RF, Lazarus DR, Kuhl K, et al. Randomized trial of endobronchial ultrasound guided transbronchial needle aspiration under general anesthesia versus moderate sedation. Am J Respir Crit Care Med 2015;191:796-803.
25. Yarmus LB, Akulian JA, Gilbert C, et al. Comparison of moderate versus deep sedation for endobronchial ultrasound transbronchial needle aspiration. Ann Am Thorac Soc 2013;10:121-6.

26. Aswanetmanee P, Limsuwat C, Kabach M, et al. The role of sedation in endobronchial ultrasound-guided transbronchial needle aspiration: Systematic review. Endosc Ultrasound 2016;5:300-6.

27. Steinfort DP, Irving LB. Patient satisfaction during endobronchial ultrasound-guided transbronchial needle aspiration performed under conscious sedation. Respir Care 2010;55:702-6.

28. Eapen GA, Shah AM, Lei X, et al. Complications, consequences and practice patterns of endobronchial ultrasound-guided transbronchial needle aspiration. Chest 2013;143:1044-53.

29. Varela-Lema L, Fernandez-Villar A, Ruano-Ravina A. Effectiveness and safety of endobronchial ultrasound-transbronchial needle aspiration: a systematic review. Eur Respir J 2009;33:1156-64.

30. Dal T, Sazak H, Tunc M, et al. A comparison of ketamine-midazolam and ketamine-propofol combinations used for sedation in the endobronchial ultrasound-guided transbronchial needle aspiration: a prospective, single-blind, randomized study. J Thorac Dis 2014; 6:742-51.

31. Abu-Hijleh M, El-Sameed Y, Eldridge K, et al. Linear probe endobronchial ultrasound bronchoscopy with guided transbronchial needle aspiration (EBUS-TBNA) in the evaluation of mediastinal and hilar pathology: introducing the procedure to a teaching institution. Lung 2013;191:109-15. 